\title{
Overview of the Wendelstein 7-X phase contrast imaging diagnostic
}

\author{
Edlund, E. M.; Porkolab, M.; Huang, Z.; Grulke, O.; Böttger, L. G.; Von Sehren, C.; Von Stechow, A.
}

Published in:

Review of Scientific Instruments

Link to article, DOI:

$10.1063 / 1.5038804$

Publication date:

2018

Document Version

Publisher's PDF, also known as Version of record

Link back to DTU Orbit

Citation (APA):

Edlund, E. M., Porkolab, M., Huang, Z., Grulke, O., Böttger, L. G., Von Sehren, C., \& Von Stechow, A. (2018). Overview of the Wendelstein 7-X phase contrast imaging diagnostic. Review of Scientific Instruments, 89(10), [10E105]. https://doi.org/10.1063/1.5038804

\section{General rights}

Copyright and moral rights for the publications made accessible in the public portal are retained by the authors and/or other copyright owners and it is a condition of accessing publications that users recognise and abide by the legal requirements associated with these rights.

- Users may download and print one copy of any publication from the public portal for the purpose of private study or research.

- You may not further distribute the material or use it for any profit-making activity or commercial gain

- You may freely distribute the URL identifying the publication in the public portal 


\section{Overview of the Wendelstein 7-X phase contrast imaging diagnostic}

E. M. Edlund, M. Porkolab, Z. Huang, O. Grulke, L.-G. Böttger, C. von Sehren, and A. von Stechow

Citation: Review of Scientific Instruments 89, 10E105 (2018); doi: 10.1063/1.5038804

View online: https://doi.org/10.1063/1.5038804

View Table of Contents: http://aip.scitation.org/toc/rsi/89/10

Published by the American Institute of Physics

\section{Articles you may be interested in}

A divertor scraper observation system for the Wendelstein $7-X$ stellarator Review of Scientific Instruments 89, 10E102 (2018); 10.1063/1.5035078

Key results from the first plasma operation phase and outlook for future performance in Wendelstein 7-X Physics of Plasmas 24, 055503 (2017); 10.1063/1.4983629

Active spectroscopy measurements of the deuterium temperature, rotation, and density from the core to scrape off layer on the DIII-D tokamak (invited)

Review of Scientific Instruments 89, 10D110 (2018); 10.1063/1.5038349

A combined phase contrast imaging and heterodyne interferometer system for multiscale fluctuation measurements (invited)

Review of Scientific Instruments 89, 10B106 (2018); 10.1063/1.5035413

Prospects of X-ray imaging spectrometers for impurity transport: Recent results from the stellarator Wendelstein 7-X (invited)

Review of Scientific Instruments 89, $10 \mathrm{G101}$ (2018); 10.1063/1.5036536

In situ wavelength calibration system for the X-ray Imaging Crystal Spectrometer (XICS) on W7-X

Review of Scientific Instruments 89, 10F107 (2018); 10.1063/1.5038809

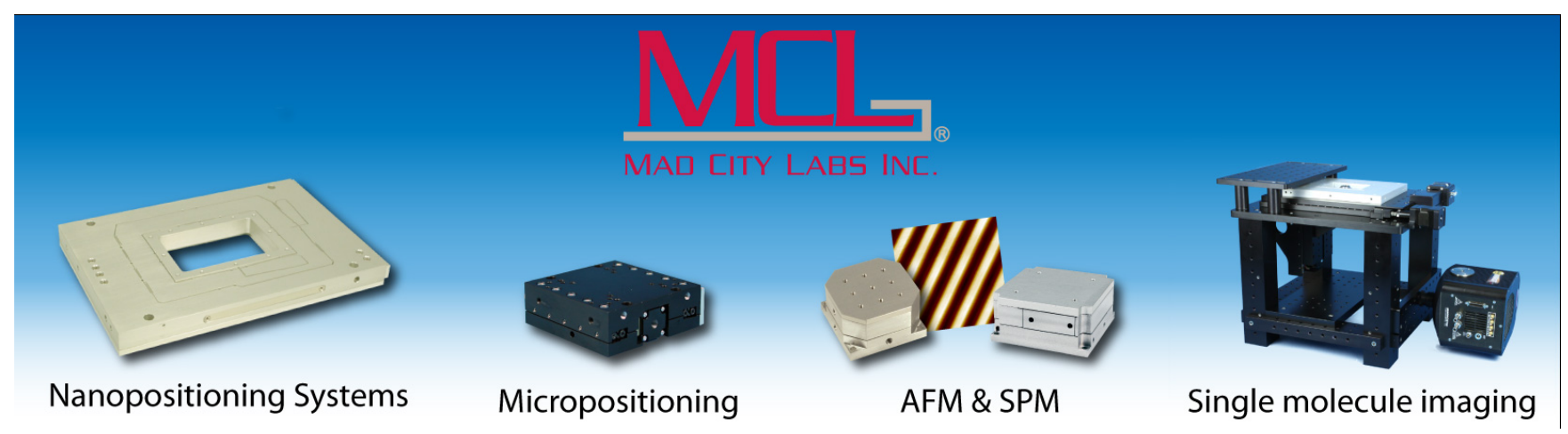




\title{
Overview of the Wendelstein 7-X phase contrast imaging diagnostic
}

\author{
E. M. Edlund, ${ }^{1, a)}$ M. Porkolab, ${ }^{2}$ Z. Huang, ${ }^{2}$ O. Grulke, ${ }^{3, b)}$ L.-G. Böttger, $\left.{ }^{3, b}\right)$ \\ C. von Sehren, ${ }^{3}$ and A. von Stechow ${ }^{3}$ \\ ${ }^{1}$ SUNY Cortland, Cortland, New York 13045, USA \\ ${ }^{2}$ MIT Plasma Science and Fusion Center, Cambridge, Massachusetts 02139, USA \\ ${ }^{3}$ Max Planck Institute for Plasma Physics, Greifswald 17491, Germany
}

(Presented 18 April 2018; received 5 May 2018; accepted 25 June 2018; published online 16 August 2018)

\begin{abstract}
A phase contrast imaging (PCI) diagnostic has been developed for the Wendelstein 7-X (W7-X) stellarator. This diagnostic, funded by the U.S. Department of Energy through the Office of Fusion Energy Sciences, is a collaboration between the Max Planck Institute for Plasmaphysics, MIT, and SUNY Cortland. The primary motivation for the development of the PCI diagnostic is measurement of turbulent fluctuations, such as the ion temperature gradient, electron temperature gradient, and the trapped electron mode instabilities. Understanding how the magnetic geometry and other externally controllable parameters, such as the fueling method and heating scheme, modify the amplitude and spectrum of turbulence is important for finding operational scenarios that can lead to improved performance at fusion-relevant temperatures and densities. The PCI system is also sensitive to coherent fluctuations, as may arise from Alfvén eigenmodes or other MHD activity, for example. The PCI method creates an image of line-integrated variations in the index of refraction. For a plasma, the image created is proportional to the line-integral of electron density fluctuations. This paper provides an overview of some key features of the hardware and the optical system and presents two examples of recent measurements from the W7-X OP1.2a experimental campaign. Published by AIP Publishing. https://doi.org/10.1063/1.5038804
\end{abstract}

\section{INTRODUCTION}

Phase contrast imaging (PCI) diagnostics have been used on a number of magnetic confinement machines, including the TCA, ${ }^{1}$ DIII-D, ${ }^{2}$ Alcator C-Mod, ${ }^{3}$ and Tokamak à Configuration Variable $(\mathrm{TCV})^{4}$ tokamaks and also on the Large Helical Device (LHD) stellarator. ${ }^{5}$ Installation of the PCI hardware on W7-X was completed in the summer of 2017, and the first operation of the diagnostic occurred in September of 2017 during the OP1.2a campaign. ${ }^{6}$

The W7-X stellarator is a large-scale fusion experiment that began operation in late $2015^{7,8}$ and recently concluded its second operational phase, OP1.2a, that ran from September 5 to December 7 of 2017. The average major and minor radii of $\mathrm{W} 7-\mathrm{X}$ are $5.5 \mathrm{~m}$ and $0.53 \mathrm{~m}$, respectively. $\mathrm{W} 7-\mathrm{X}$ is of the Helias line of stellarators, with a nearly quasi-isodynamic magnetic field geometry at high $\beta$ (where $\beta \sim p / 4 \mu_{0} B^{2}$ ), and was designed with consideration given to seven engineering and physics constraints, including low neoclassical losses of particles and energy and low bootstrap current. ${ }^{9}$ The ability to vary the coil currents provides external control over the mirror ratio, rotational transform, and the position of the magnetic axis. One of the major goals of the $\mathrm{W} 7-\mathrm{X}$ research program is to understand the impact of the magnetic field configuration on

Note: Paper published as part of the Proceedings of the 22nd Topical Conference on High-Temperature Plasma Diagnostics, San Diego, California, April 2018.

a) eric.edlund@cortland.edu

b) Also affiliated with the Technical University of Denmark, Kongens Lyngby, Denmark. global performance through its influence on both neoclassical and turbulent transport. One of the primary goals of the W7-X PCI diagnostic is to help assess the nature of turbulence and its impact on global transport, especially as it relates to the magnetic configuration.

The ISS $_{04}$ scaling, which describes the multi-parameter scaling for stellarator energy confinement times, indicates that high-performance plasmas in W7-X should greatly exceed the performance of all previous stellarators and approach that of similarly sized tokamaks. ${ }^{10}$ Analysis of experiments from the W7-X OP1.1 campaign indicates performance congruent with the $\mathrm{ISS}_{0} 4$ scaling ${ }^{8}$ though these energy confinement times are still about an order of magnitude smaller than the best tokamak $\mathrm{H}$-mode energy confinement times.

One of the outstanding points of uncertainty in our predictive capability, applying to higher performance scenarios in W7-X and upcoming next-generation devices like ITER, is how turbulent transport will scale as we move to larger devices and higher temperatures and densities. A variety of turbulent mechanisms, including the ion temperature gradient (ITG) instability, the electron temperature gradient (ETG) instability, and the trapped electron mode (TEM) instability, are expected to modify the transport and energy confinement properties of these plasmas. ${ }^{11,12}$ This is an important area of research to address in anticipation of future burning plasma experiments that will be dominated by electron-heating. Electromagnetic modes, such as kinetic ballooning modes ${ }^{13}$ and Alfvén eigenmodes, ${ }^{14}$ may also modify the transport of the bulk plasma or energetic components. The role of the magnetic field geometry on turbulence and instabilities, and therefore the 
degree of external control over transport, is a subject of much interest.

This paper describes the principle of the phase contrast method in Sec. II, briefly describes some of the main hardware and design features of the W7-X PCI system in Sec. III, presents a few measurements from the OP1.2a campaign in Sec. IV, and concludes with an outlook to future experiments and system upgrades in Sec. V.

\section{THE PHASE CONTRAST METHOD}

The PCI diagnostic is in essence a small-angle scattering system, where electron density perturbations induce variations in the index of refraction that diffract the incident light as it passes through the plasma. ${ }^{15}$ The measured signal is an image of the variations in the index of refraction of the probed medium. For a plasma, the intensity of the image is proportional to the line-integral of electron density fluctuations. The line-integrated nature of the signal is both a challenge and a strength of the PCI diagnostic. Extracting information on the 3D nature of fluctuations is difficult and requires detailed comparisons to other measurements or simulations and can be aided by the use of a spatial filter that localizes signals according to the magnetic pitch angle and a comparison to numerical predictions. On the other hand, the PCI diagnostic, being generally sensitive to density fluctuations from any source and across all regions of the plasma along the beam path, can simultaneously measure broadband fluctuations from the plasma edge and coherent mode activity, such as Alfvén eigenmodes that may exist near the plasma core. Regardless of the source of the diffraction, the image intensity at the detector is proportional to the line-integral of the electron density fluctuations presenting spatial variations perpendicular to the beam path. ${ }^{15}$

In the phase contrast method, electron density fluctuations are equivalent to a phase grating for the incident light. Most of the power is carried forward by the direct $(m=0)$ component of the beam, and the information regarding the spatial structures and amplitudes of the fluctuations is carried by the first-order diffracted $(m= \pm 1)$ components. By comparing the diffracted components that traverse the same plasma region to the direct component, the effect of the mean density is effectively absent in the image and the output signal depends only on the plasma fluctuations. The amplitude of the diffracted signal $(\Delta)$ is proportional to the line integral of the density fluctuations along the beam path, that is,

$$
\Delta=E_{0} r_{e} \lambda \int \tilde{n}_{e} \mathrm{~d} z,
$$

where $E_{0}$ is the incident electric field amplitude of the laser beam, $r_{e} \approx 2.8 \times 10^{-15} \mathrm{~m}$ is the classical electron radius, $\lambda=10.6 \mu \mathrm{m}$ is the laser wavelength, and the integral over the electron density fluctuations is taken along the beam path through the plasma. As an example of the fluctuation amplitude, a W7-X plasma with $n_{e} \approx 1 \times 10^{19} \mathrm{~m}^{-3}$ and fluctuations with $\tilde{n}_{e} / n_{e} \approx 10^{-2}$ that are present over one quarter of the path through the plasma (roughly $0.25 \mathrm{~m}$ ) would have $\Delta / E_{0} \sim 10^{-3}$.

Critical to the analysis of image formation in the phase contrast method is the recognition that the diffracted components have a mean phase of $\pi / 2$ relative to the direct beam. This phase shift arises because the diffracted components carry the information about the plasma "phase grating" which acts either to advance or retard the wave phase and hence is necessarily out of phase with the direct beam. In the regime of weak diffraction, an image created by combining the direct and diffracted components would not show any features representative of the plasma fluctuations as the fluctuations only modify the phase and have no effect on the amplitude of the incident light. Therefore, imaging of fluctuations requires some manipulation of the relative phase between the direct and diffracted components. This modification of phase is performed by the phase plate. Located at a focal plane of the optical system, the phase plate takes advantage of the spatial separation of the Fourier components comprising the phase grating presented by the plasma. The phase plate is in essence a $2^{\prime \prime}$ diameter flat mirror (gold plated) with a $1.0 \mathrm{~mm}$ wide groove of depth $\lambda / 8$ spanning a diameter. The direct component is focused onto the groove and, having traveled into and back out of the groove, travels a total extra distance of $\lambda / 4$ for a phase shift of $\pi / 2$. The optic substrate, being $\mathrm{ZnSe}$, has a reflection coefficient of $R \approx 0.28$ and reduces the amplitude of the direct component. Thus, having advanced the phase of the direct component by $\pi / 2$, the components at the detector sum to create an intensity given by

$$
I_{P C I}=\left|i R^{1 / 2} E_{0}+i \Delta\right|^{2} \approx R E_{0}^{2}+2 R^{1 / 2} E_{0} \Delta .
$$

The linearity of the intensity in the diffracted signal $(\Delta)$ means that the measurement is linear in the line-integral of the electron density fluctuations. The fraction of the signal attributed to the fluctuations is $2 \Delta / R^{1 / 2} E_{0}$, which is enhanced by a factor of $R^{-1 / 2} \approx 1.89$ over a perfectly reflective phase plate. The detector preamplifiers apply a high-pass filter and eliminate the DC component $\left(E_{0}^{2}\right)$ of the image, yielding an output signal that is simply proportional to $\Delta$, modified by the frequency response of the detector and preamplifier network. These effects are discussed more in Sec. III.

A spatial masking device, not yet implemented, will localize fluctuations through the relationship between the magnetic pitch angle within the plasma and the angular position of scattered signals at a focal plane. A more detailed analysis of the optical system and its sensitivity to the entire range of wavenumbers will be provided in a subsequent publication.

\section{OVERVIEW OF THE OPTICAL SYSTEM AND HARDWARE}

Spatial constraints in the W7-X torus hall and through the torus require that the laser and detector be situated at different locations on separate optical tables. The $\mathrm{CO}_{2}$ and HeNe lasers together with the optics to condition and expand the beams are located on the transmitting table, shown in Fig. 1, in the second basement level of the W7-X torus hall. The detector together with the phase plate and imaging optics is located on the receiving table, shown in Fig. 2, in the first basement level of the torus hall. Each table is covered by a light-tight enclosure, and the entire beam path is enclosed by a beam duct. A total path length of approximately 22 meters separates the transmitting and receiving tables. 


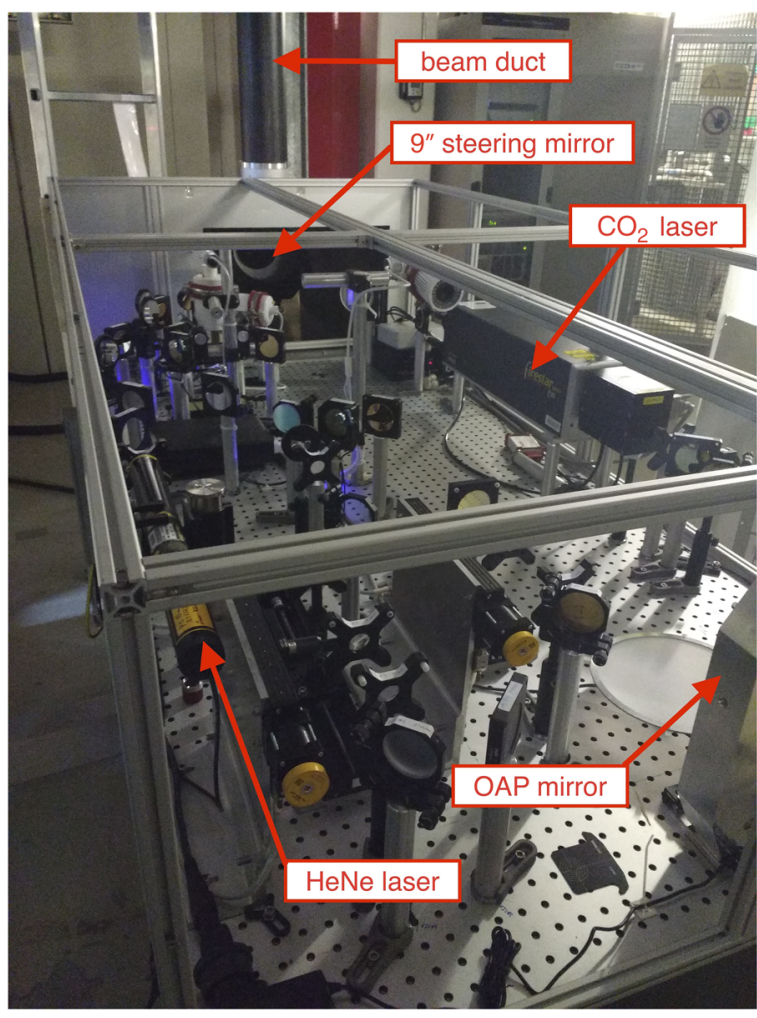

FIG. 1. Photo of the receiving table showing the $\mathrm{CO}_{2}$ laser, $\mathrm{HeNe}$ laser, the 9" diameter off-axis-parabolic (OAP) mirror, the first 9 " diameter steering mirror, and the beam duct up to the first mirror box.

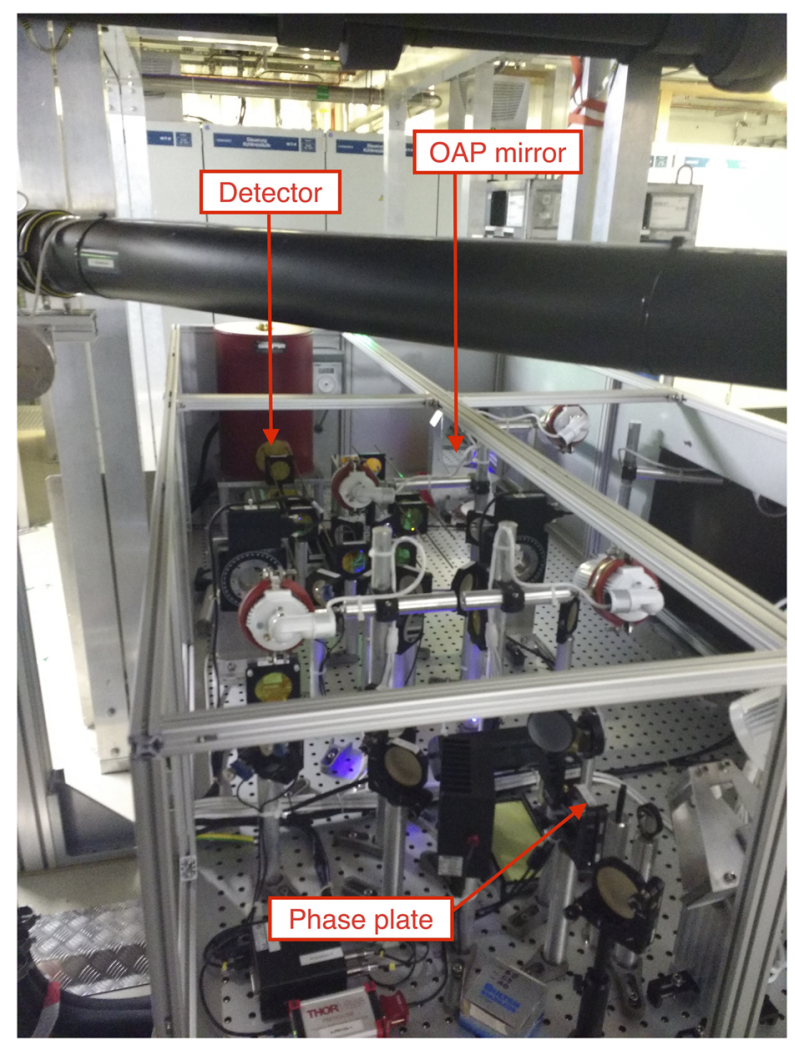

FIG. 2. Photo of the receiving table showing the 9 " diameter OAP mirror, the detector, and the phase plate. The black tube passing over the top of the table is the beam duct for the Thomson scattering system.
The light source for the PCI system is a $100 \mathrm{~W} \mathrm{CO}_{2}$ industrial-quality laser operating at a wavelength of $10.6 \mu \mathrm{m}$. A HeNe laser (632.8 $\mathrm{nm}$ wavelength) is combined with the $\mathrm{CO}_{2}$ beam to assist with alignment for safe operation during open access to the torus hall. Optics on the transmitting table combine the $\mathrm{HeNe}$ and $\mathrm{CO}_{2}$ laser beams, and, once combined, the combined beams are expanded with a telescope comprised of two off-axis parabolic (OAP) mirrors with a magnification of $M=80^{\prime \prime} / 6^{\prime \prime}=13.3$. A typical beam size out of the telescope during the OP1.2a campaign was about $8.0 \mathrm{~cm}$.

A series of seven $9^{\prime \prime}$ diameter steering mirrors deliver the beams from the transmitting table to the receiving table. The first sends the beam from the transmitting table vertically up along the inside of the torus toward the first window. Two mirror boxes, one located adjacent to each port window, house additional mirrors that redirect the beam into and out of the W7-X cryostat. Two steering mirrors within the first mirror box redirect the beam horizontally in front of the port window and then deflect it upward at the required $18^{\circ}$ angle, with respect to the horizontal. Two mirrors in the second mirror box steer the beam first horizontally and then down to the receiving table. The two final $9^{\prime \prime}$ steering mirrors direct the beam onto the $9^{\prime \prime}$ diameter OAP mirror for focusing onto the phase plate. Following the phase plate, the beam is collimated by a second OAP mirror, forming a telescope with a magnification of $M=6^{\prime \prime} / 80^{\prime \prime}=0.075$. After the second OAP, the collimated beam passes through two $2^{\prime \prime}$ diameter, $16^{\prime \prime}$ focal-length $\mathrm{ZnSe}$ lenses that are separated by $32^{\prime \prime}$. A wavenumber filter, for selecting scattered components at particular angles, can be placed at the focal plane located between these two lenses. Three final $\mathrm{ZnSe}$ lenses (focal lengths of $5^{\prime \prime}, 8.5^{\prime \prime}$, and $16^{\prime \prime}$ ) form a detector telescope and create an image at the detector. The latter two lenses are mounted on linear stages that allow the diagnostic operators to remotely adjust the image magnification in the range of approximately $1-5$. The net magnification of the system is then equal to the product of the magnification from the OAP mirrors (0.075) and the magnification of the detector imaging telescope and is in the range of $0.075-0.375$, approximately.

The detector is a linear array of $32 \mathrm{HgCdTe}$ elements, each with a width of $0.5 \mathrm{~mm}$, operating in the photo-conductive mode. The detector is cooled with liquid nitrogen to reduce thermal noise. The frequency response of the detector has been calibrated by measuring the element output voltage in response to a modulated signal from an array of LEDs. An example of the frequency response curve for a single element is shown in Fig. 3.

The detectable wavenumber range depends on the beam diameter and phase plate geometry in the lower limit and the net system magnification and the width of the detector array size in the upper limit. At a typical final magnification of 3 (net magnification of 0.225), the distance in the plasma imaged over the 32 detector elements is about $7.1 \mathrm{~cm}$. This provides a typical $k_{\max } \approx 14 \mathrm{~cm}^{-1}$. The lower limit to the wavenumber range is set by the requirement that diffracted components fall outside the groove of the phase plate, which gives $k_{\min } \approx 1.5 \mathrm{~cm}^{-1}$. For an $8.0 \mathrm{~cm}$ beam diameter in the plasma and a net magnification of $3.0 \times 0.075=0.225$, the $8.0 \mathrm{~cm}$ beam diameter is imaged onto the detector with a diameter of $1.8 \mathrm{~cm}$, 


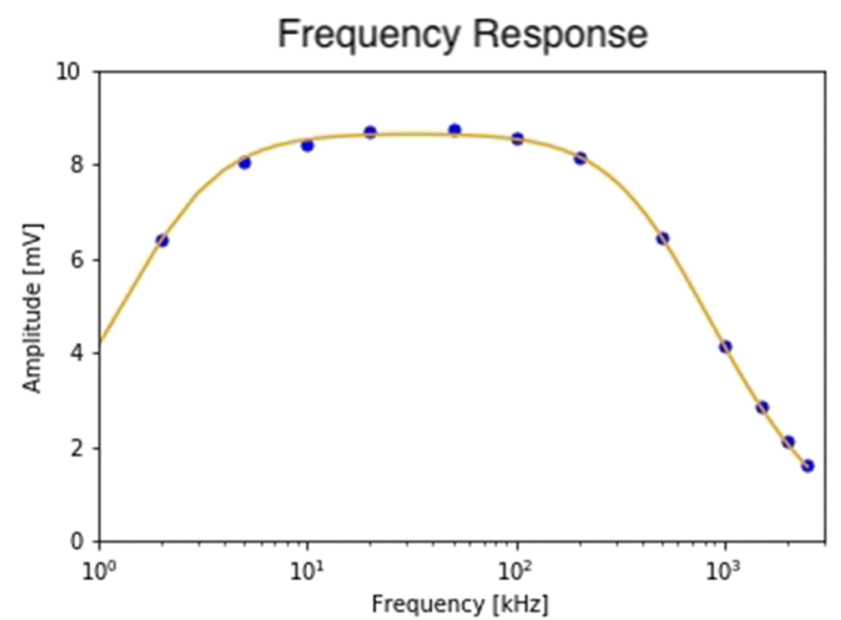

FIG. 3. Calibration curve for detector channel 1 . This system can be modeled as a bandpass filter with an approximate $2 \mathrm{kHz}$ high-pass frequency and an approximate $572 \mathrm{kHz}$ low-pass frequency. The blue dots are measurements of the amplitude response inferred from measurements of sinusoidally modulated light from an array of LEDs, and the orange (solid) curve is the bandpass function.

corresponding to a channel-to-channel spacing projected onto the plasma of about $0.25 \mathrm{~cm}$. This magnification range is ideally suited to detecting TEM turbulence with $k_{\theta} \rho_{s} \sim 1$, corresponding to a fluctuation wavenumber of about $2.5 \mathrm{~cm}^{-1}$ and a wavelength of approximately $2.5 \mathrm{~cm}$. The ITG and ETG fluctuations, occurring at length scales an order of magnitude smaller and larger than the TEM, respectively, are somewhat outside of the range for optimal detection with the PCI system at the typical magnification. Additionally, these modes can be distinguished by the direction of propagation in the plasma frame, with ITG rotating in the ion-diamagnetic direction, and the ETG and TEM modes rotating in the electron diamagnetic direction. Simulations of ITG $^{12}$ and TEM ${ }^{11}$ turbulence indicate that these modes should have substantially larger amplitude on the outboard side of the plasma, compared to the inboard side, and therefore, it is expected that the PCI diagnostic will measure an asymmetric k-spectra that is representative of the direction of propagation.

An ultrasonic sound wave at a frequency of $43 \mathrm{kHz}$ $\left(\lambda \approx 0.79 \mathrm{~cm}, k \approx 7.9 \mathrm{~cm}^{-1}\right)$ is launched in air in the second mirror box. Measurement of the sound waves serves two purposes. First, the known wavelength in air provides a check of the imaging system magnification. Second, if the density perturbations from these sound waves are known in absolute units and the spatial structure of the waves is known accurately, then the change in index of refraction in air can be related to the change in index of refraction of the plasma, allowing absolute density fluctuation levels to be determined.

\section{SURVEY OF MEASUREMENTS}

Typical plasma parameters for OP1.2a plasmas had $T_{e}$ in the range of 2-4 keV, $T_{i}$ in the range of $1-2 \mathrm{keV}$, and $n_{e}$ in the range of $2-4 \times 10^{19} \mathrm{~m}^{-3}$, in both helium and hydrogen plasmas. Heating in the OP1.2a campaign consisted exclusively of up to $8.5 \mathrm{MW}$ of X-mode electron cyclotron resonance heating (ECRH). During the OP1.2a campaign, the PCI diagnostic

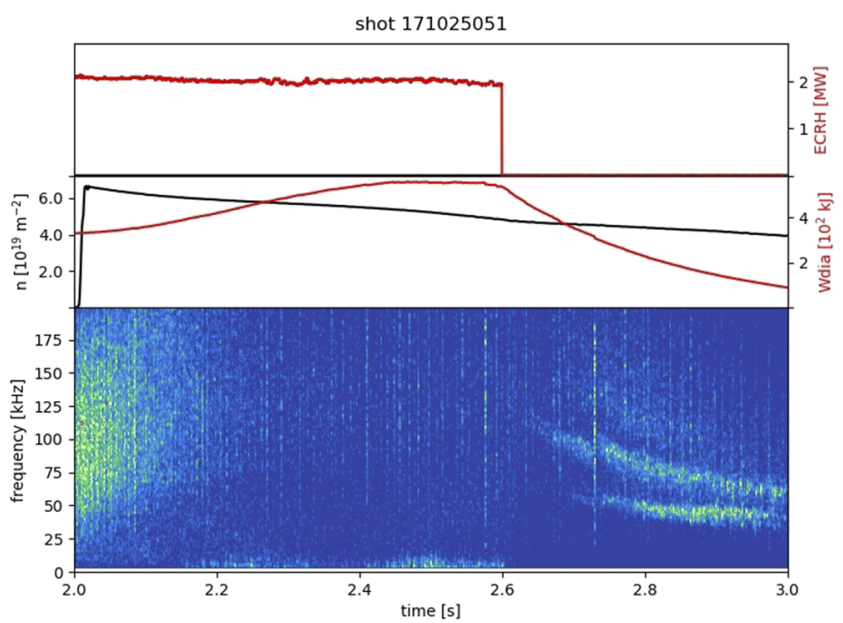

FIG. 4. Frequency spectrogram showing a series of down-chirping modes near the end of a plasma experiment, perhaps associated with a rotating magnetic island that is slowing as the plasma density decreases.

measured a wide range of phenomena, including broadband spectra that exhibit variations with the magnetic configuration, fluctuations in the after-glow phase after ECRH is terminated, and coherent fluctuations likely from Alfvén eigenmodes.

Precise identification of the driving mechanisms of turbulence requires careful analysis and comparisons with other diagnostics. While such studies are underway, no conclusive results are yet available. In lieu of presenting measurements of broadband turbulence, we focus here instead on a select set of coherent modes that illustrate the capability of the PCI diagnostic. Figure 4 shows a set of semi-coherent modes that may be due to a rotating magnetic island whose rotation slows, and hence the measured frequency decreases, in the after-glow phase.

Figure 5 shows a series of modes persisting over almost $1.5 \mathrm{~s}$ that are thought to be Alfvénic in nature due to the strong correlation with the Alfvén frequency and no significant correlation with temperature. The absence of similar signals on the magnetics suggests that these modes are located deep within

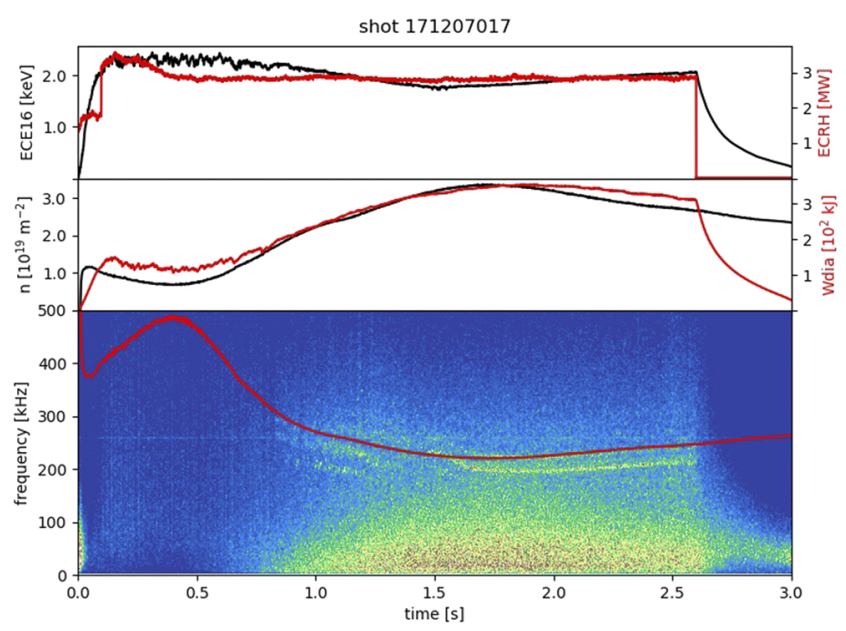

FIG. 5. A series of coherent modes near $200 \mathrm{kHz}$ appear to have an Alfvénic nature based on their scaling with density. The red overlay shows the scaled change in $n_{e}^{-1 / 2}$. 
the plasma core. It has been previously shown that the spatial structure and frequency of some Alfvénic modes are sensitive to the plasma equilibrium and can therefore provide additional information regarding properties of the deep core. ${ }^{16}$ It is somewhat surprising that Alfvénic modes could be driven in plasmas that are purely electron heated with ECRH and thus absent of any energetic-ion component. However, similar modes were observed in the W7-X OP1.1 campaign and it was speculated that they are excited by the thermal electron population or energetic trapped electrons. ${ }^{17}$ In some prior experiments in the COMPASS ${ }^{18}$ and Alcator C-Mod ${ }^{19}$ tokamaks, Alfvénic activity was correlated with fast-electrons created by lowerhybrid waves. Somewhat related are that studies from Helical Symmetric Experiment (HSX) showed that a population of energetic electrons from ECRH could excite acoustic modes ${ }^{20}$ though we anticipate that the frequency in $\mathrm{W} 7-\mathrm{X}$ is far too high to be relevant to the observations reported here.

\section{CONCLUSIONS}

The W7-X PCI diagnostic is providing measurements of coherent modes and turbulent fluctuations. Coherent modes that exhibit a strong correlation with the Alfvén frequency are likely Alfvén eigenmodes. These measurements may provide insight into the spectrum of weakly damped Alfvén modes that will be of greater concern once an energetic-ion population is present. While the analysis of the turbulent fluctuations is ongoing and in the early stages of vetting, some initial observations on turbulent fluctuations in different magnetic configurations with similar plasma values suggest significant changes in the fluctuation levels and spectral shape.

Future upgrades for the PCI system include a second detector with an identical optical system. By placing a spatial filter on each one of these beam lines, the PCI diagnostic will be able to simultaneously localize fluctuations from two radial regions within the plasma. The goal of this system upgrade is to provide constraints on radial correlation lengths in addition to the poloidal structures that are regularly measured by a single detector array. Other hardware upgrades underway include an improved sound wave source for calibration, remotely positionable optics that will allow for modifications to the beam size, and an improved range of magnifications.

\section{ACKNOWLEDGMENTS}

This work is sponsored by the U.S. Department of Energy, Office of Fusion Energy Sciences, under Grant No. DESC0014229. This work has been carried out within the framework of the EUROfusion Consortium and has received funding from the Euratom research and training programme 2014-2018 under Grant Agreement No. 633053. The views and opinions expressed herein do not necessarily reflect those of the European Commission.

${ }^{1}$ H. Weisen, Rev. Sci. Instrum. 59, 1544 (1988).

${ }^{2}$ S. Coda and M. Porkolab, Rev. Sci. Instrum. 63, 4974 (1992).

${ }^{3}$ L. Lin, E. M. Edlund, M. Porkolab, Y. Lin, and S. J. Wukitch, Rev. Sci. Instrum. 77, 10E918 (2006).

${ }^{4}$ A. Marinoni, S. Coda, R. Chavan, and G. Pochon, Rev. Sci. Instrum. 77, 10E929 (2006).

${ }^{5}$ C. A. Michael and K. Tanaka, Rev. Sci. Instrum. 77, 10E923 (2006).

${ }^{6}$ E. M. Edlund, M. Porkolab, O. Grulke, A. von Stechow, and L.-G. Böttger, Bull. Am. Phys. Soc. 62, 90 (2017), abstract available at http://meetings. aps.org/link/BAPS.2017.DPP.CP11.53.

${ }^{7}$ H.-S. Bosch et al., Nucl. Fusion 57, 116015 (2017)

${ }^{8}$ R. C. Wolf et al., Nucl. Fusion 57, 102020 (2017).

${ }^{9}$ G. Grieger et al., Phys. Fluids B 4, 2081 (1991).

${ }^{10}$ A. Dinklage et al., Nucl. Fusion 47, 1265 (2007).

${ }^{11}$ J. H. E. Proll, H. E. Mynick, P. Xanthopoulos, S. A. Lazerson, and B. J. Faber, Plasma Phys. Controlled Fusion 58, 014006 (2016).

${ }^{12}$ P. Xanthopoulos, H. E. Mynick, P. Helander, Y. Turkin, G. G. Plunk, F. Jenko, T. Görler, D. Told, T. Bird, and J. H. E. Proll, Phys. Rev. Lett. 113, 155001 (2014).

${ }^{13}$ B. F. McMillan and R. L. Dewar, Nucl. Fusion 46, 477 (2006).

${ }^{14}$ Y. I. Kolesnichenko, Y. V. Yakovenko, A. Weller, A. Werner, J. Geiger, V. V. Lutsenko, and S. Zegenhagen, Phys. Rev. Lett. 94, 165004 (2005).

${ }^{15}$ M. Porkolab, C. Rost, N. Basse, J. Dorris, E. M. Edlund, L. Lin, Y. Lin, and S. J. Wukitch, IEEE Trans. Plasma Sci. 34, 229 (2006).

${ }^{16}$ E. M. Edlund, M. Porkolab, G. J. Kramer, L. Lin, Y. Lin, and S. J. Wukitch, Phys. Rev. Lett. 102, 165003 (2009).

${ }^{17}$ T. Windisch, A. Krämer-Flecken, J. L. Velasco, A. Könies, C. Nührenberg, O. Grulke, T. Klinger, and W7-X Team, Plasma Phys. Controlled Fusion 59, 105002 (2017).

${ }^{18}$ M. Valovic, B. Llyoyd, K. G. McClements, C. D. Warrick, S. J. Fielding, A. W. Morris, T. Pinfold, and H. R. Wilson, Nucl. Fusion 40, 1569 (2000).

${ }^{19}$ J. A. Snipes, R. R. Parker, P. E. Phillips, A. Schmidt, and G. Wallace, Nucl. Fusion 48, 072001 (2008).

${ }^{20}$ C. B. Deng, D. L. Brower, B. N. Breizman, D. A. Spong, A. F. Almagri, D. T. Anderson, F. S. B. Anderson, W. X. Ding, W. Guttenfelder, K. M. Likin, and J. N. Talmadge, Phys. Rev. Lett. 103, 025003 (2009). 